\title{
CINCO NUEVOS REGISTROS DE LIQUENES MARINOS PARA CHILE
}

\section{FIVE NEW RECORDS OF MARINE LICHENS FROM CHILE}

\author{
Iris Pereira \& Waleska Torres \\ Instituto de Biología Vegetal y Biotecnología, Universidad de Talca, Casilla 747, Talca. \\ ipereira@utalca.cl
}

\section{RESUMEN}

Cinco especies de líquenes marinos son documentadas por primera vez en Chile:Arthopyrenia saxicola Massal., Caloplaca carphinea (Fr.) Jatta, C. thallincola (Wedd.) Du Rietz, Pertusaria rupicola (Fr.) Harm. y Xanthoria ectaneoides (Nyl.) Zahlbr. Dichos taxones fueron hallados en la Bahía de Constitución, Chile Central. Para cada taxón, se entrega una descripción basada en el material estudiado consistente en la morfología externa del talo y las características morfológicas internas de las estructuras reproductoras de cada especie. También se incluyen las reacciones químicas del talo y de algunas estructuras reproductoras, cuando el taxón lo amerita. Además se adjuntan fotografías a color de los talos de las especies estudiadas.

Palabras claves: Chile Central, litoral costero, orilla rocosa.

\section{ABSTRACT}

Five marine lichen species are reported for the first time in Chile: Arthopyrenia saxicola Massal., Caloplaca carphinea (Fr.) Jatta, C. thallincola (Wedd.) Du Rietz, Pertusaria rupicola (Fr.) Harm. and Xanthoria ectaenoides (Nyl.) Zahlbr. These were found at Bahía de Constitución, Central Chile. Descriptions of the material cover the external morphology of the thallus and the internal morphological characters of the reproductive structures. Colour photos of the thallus of all species are included, plus for some taxa we also describe chemical reactions of the thallus and of some reproductive structures.

KEYwords : Central Chile, littoral zone, rocky shores.

\section{INTRODUCCION}

El litoral costero del norte y centro de Chile continental están siendo sometidos a diversos efectos antrópicos, los cuales podrían tener repercusiones impredecibles sobre la diversidad de líquenes marinos (Follmann 1995). Estos ecosistemas están siendo alterados mediante la construcción habitacional, la frecuentación turística del litoral y la contaminación de las aguas marinas mediante la eliminación de diversos contaminantes en aguas continentales que finalmente desembocan al mar (CONAMA 2005, Cornejo 2003).

En Chile, son pocos los trabajos que incluyen información acerca de la morfología externa del talo y características morfológicas internas de las estructuras reproductoras de estos líquenes, lo cual no siempre facilitan en un corto plazo la determinación taxonómica de ellos. Entre los trabajos que incluyen este tipo de información se pueden señalar las escasas revisiones de ciertos taxones de la familia Roccellaceae: Dirina Fr. y Roccellina Tehler (Tehler 1983), Roccella Tehler (Follmann 1964, 1968a) y Arthoniaceae: Arthonia Ach. (Follmann 1968b), cuya representación es destacable en el litoral costero de nuestro país.

Este artículo describe la morfología externa del talo y los caracteres morfológicos internos de las estructuras reproductoras de cinco líquenes del litoral costero de Chile central. Ninguno de estos 
taxones ha sido citado previamente en Chile (Galloway \& Quilhot 1998, Feuerer 2004) y por ende este artículo incrementa el conocimiento de la diversidad liquénica en el país.

\section{MATERIALES Y METODOS}

\section{AREA DE ESTUDIO}

El área de estudio corresponde a dos sectores del litoral costero en la Bahía de Constitución, en la región del Maule, Chile central. Los sectores son conocidos vulgarmente como Playa de Los Gringos (35 19 '35' ' S, $72^{\circ} 25^{\prime} 43^{\prime \prime}$ 'O) y Playa Vega Los Patos (35'19'43', S, 72 $26^{\circ}$ '02', O). El sustrato rocoso de ambos sectores es principalmente granítico, aunque también existen rocas metamórficas. El clima en el área de estudio es de tipo mediterráneo marítimo (Santibáñez et al. 1993).

\section{Colecta del Material ESTUdiado}

Para estudiar la morfología de las especies, se dispuso de material liquénico proveniente de dos sectores localizados en la Bahía de Constitución. Los líquenes fueron colectados sobre sustratos rocosos dentro de cada una de las zonas descritas por Santelices (1989) para el litoral costero de Chile Central. En cada sitio se establecieron tres transectos verticales entre la zona litoral y supralitoral. Los transectos verticales estuvieron distanciados por $5 \mathrm{~m}$. Dentro de cada zona de los tres transectos preestablecidos, se colectaron cinco muestras al azar.

\section{DETERMINACIÓN TAXONÓMICA}

Las determinaciones taxonómicas se basaron en: Clauzade \& Roux (1985), Poelt (1969), Poelt \& Vezda (1977), Wirth (1980) y la nomenclatura sigue a Esslinger \& Egan (1995). Las muestras colectadas han sido depositadas en el Herbario de la Universidad de Talca, Talca. Chile.

\section{Morfología del talo}

Se registraron los caracteres vegetativos externos de los talos, considerando: el tipo morfológico, la textura y el color, así como también las reacciones químicas del cortex superior del talo y la médula. Para analizar las reacciones químicas de ambas capas, se realizaron cortes perpendiculares al talo a mano alzada utilizando una hoja de afeitar.
Los cortes fueron montados en portaobjetos sobre los cuales se aplicaron los diferentes reactivos químicos para detectar su coloración. En estas capas se aplicaron las siguientes reacciones químicas: $\mathrm{K}(\mathrm{KOH}), \mathrm{C}$ (Hipoclorito de $\mathrm{Na}$ ), $\mathrm{KC}$ combinación K seguido de C, P (parafenilendiamina) (White \& James 1985). Las reacciones colorimétricas fueron observadas mediante una lupa binocular Kyowa en el momento justo de la aplicación de cada reactivo sobre los diferentes cortes realizados para cada tinción, excepto para $\mathrm{KC}$, donde se aplicó primeramente $\mathrm{K}$ y posteriormente $\mathrm{C}$.

AnAtomia de los ascocarpos

Para el estudio de los apotecios y peritecios de las especies señaladas, se midió el diámetro de 10 de ellos mediante una lupa binocular Kyowa equipada con una reglilla graduada adaptada al ocular. Las mediciones se realizaron con un aumento de $400 \mathrm{X}$.

Para estudiar la estructura anatómica de los ascocarpos, se realizaron cortes longitudinales radiales. Luego los cortes fueron montados en fase acuosa depositada sobre los portaobjetos, cubiertos más tarde con cubreobjetos para finalmente analizar sus estructuras internas como: altura, color del tecio o himenio, del epitecio y el hipotecio y algunas reacciones químicas de éstas, de acuerdo al taxón en cuestión. Se analizó además el tipo, color y medidas de paráfisis, ascos y esporas.

Todas las mediciones se realizaron con una reglilla graduada, como se menciona antes, con aumento $400 \mathrm{X}$ ó $1000 \mathrm{X}$, dependiendo del tamaño de las esporas. Los dibujos fueron realizados con el apoyo de una cámara clara. Para visualizar las diferentes reacciones calorimétricas, los reactivos fueron aplicados por uno de los bordes del cubreobjetos utilizando en cada caso una pipeta Pasteur, siempre y cuando el taxón lo ameritara.

Las especies se ordenan alfabéticamente dentro de cada familia. Se entrega una descripción morfológica del talo de cada taxón, de los ascocarpos y reacciones químicas de ambos. Se incluye además la distribución conocida a nivel mundial. Se adjuntan dibujos de esporas de cada especie y fotografías que ilustran la morfología externa de los talos. 


\section{RESULTADOS}

ARTHOPYRENIACEAE

(Aptroot \& Sipman 2001, Coppins 2002)

\section{Arthopyrenia saxicola Massal. (Figs. 1 y 6)}

Talo crustáceo, fisurado-areolado, de aspecto pulverulento, grisáceo a negruzco. Ficobionte: Trentepohlia (L.) Martius. Médula anaranjada. Peritecios de 0,1-0,2 mm de diámetro. Involucrelo negro cubriendo un tercio del excípulo. Excípulo incoloro. Paráfisis simples. Ascos cilíndricos, octosporados. Esporas claviformes, con tres septos transversales, incoloras, de 22,4-28 x 6,4-7,2 $\mu \mathrm{m}$.

Reacciones Químicas: Cortex: K-, C-, KC-, P-. Médula: K-, C-, KC-, P-.

Distribución mundial: China (Aptroot \& Sipman 2001), Gran Bretaña e Irlanda (Coppins 2002).

Material estudiado: CHILE, VII Región, Prov. Talca, Constitución, Playa Vega Los Patos, 28-IV2002, W. Torres, en superficies rocosas planas de rocas graníticas de la zona supralitoral, $N^{\circ} 410$ (Herbario Universidad de Talca).

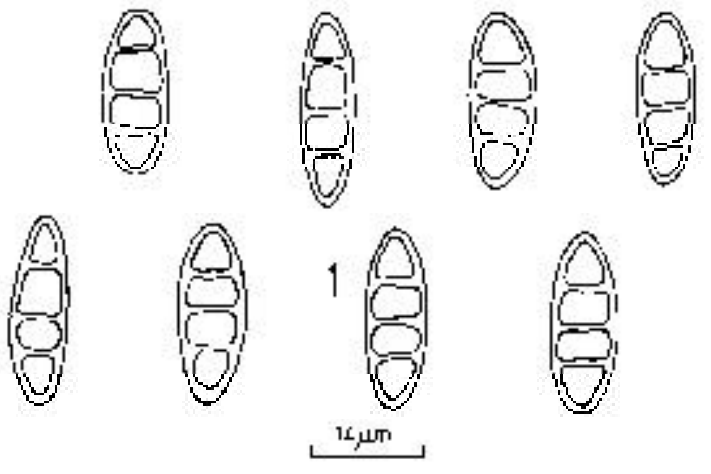

FIGURA 1. Arthopyrenia saxicola esporas.

Pertusariaceae

(Archer 1997, Archer \& Elix 1993)

Pertusaria rupicola (Fr.) Harm. (Figs. 2 y 7 )

Talo crustáceo, fisurado areolado, grisáceo a blanquecino-verdoso. Fotobionte: Trebouxia Puym. Apotecios en verrugas, de 0,75-0,8 mm. Tecio incoloro, amarillento claro, de 500-550 $\mu \mathrm{m}$ de altura.
Epitecio pardo claro. Hipotecio pardo amarillento. Ascos octosporados. Paráfisis simples, delgadas. Esporas elipsoidales, incoloras, 62-92 x 36-47,3 $\mu \mathrm{m}$, con una pared lisa, gruesa, de 3,4-6,8 $\mu \mathrm{m}$.

REACCIONES QUímicas: Cortex superior del talo: K+ amarillo, P-.

Distribución mundial: Región mediterránea y Atlántica (Clauzade \& Roux 1985); Marruecos (Egea 1996).

Material estudiado: CHILE, VII Región, Prov. Talca. Constitución, Playa Vega Los Patos. 28-IV2002. W. Torres, en la zona supralitoral, $\mathrm{N}^{\circ} 416$ (Herbario Universidad de Talca).

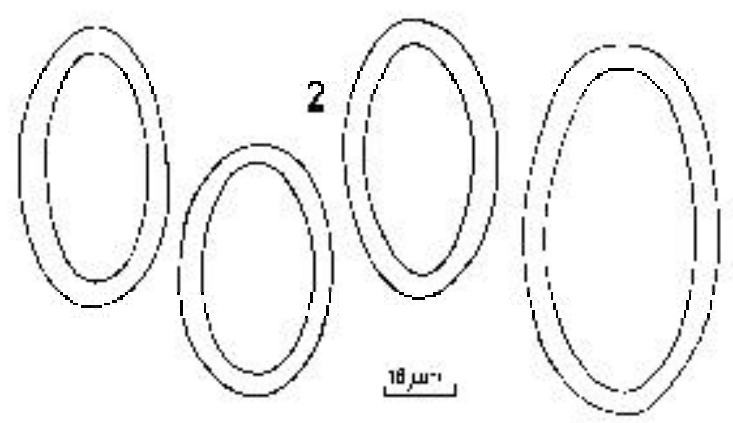

Figura 2. Pertusaria rupicola esporas.

Teloschistaceae

(Galloway \& Quilhot 1998, Malcolm \& Galloway 1997)

Caloplaca carphinea (Fr.) Jatta (Figs. 3 a, b, c y 8) Talo crustáceo, areolado, verde-amarillento, con hipotalo que se observa como una línea negra extendiéndose por fuera del límite del talo. Fotobionte: Trebouxia. Médula blanca. Apotecios lecanorinos rojo-anaranjados, de 0,1-0,7 mm de diámetro. Tecio incoloro, de 90-100 $\mu \mathrm{m}$ de altura. Epitecio anaranjado. Hipotecio incoloro. Ascos octosporados, Paráfisis en su mayoría simples, con ápices levemente abultados, a veces ramificadas y anastomosadas. Esporas polariloculares, incoloras, 12-15,5 x 5,6-8 $\mu \mathrm{m}$, con un septo ocupando un tercio del largo, de 4,8-5,6 $\mu \mathrm{m}$.

ReaCCIONeS QUímicas: Cortex superior $\mathrm{C}+\mathrm{y} \mathrm{KC}+$ anaranjado. Apotecios y epitecio $\mathrm{K}+$ púrpura. 


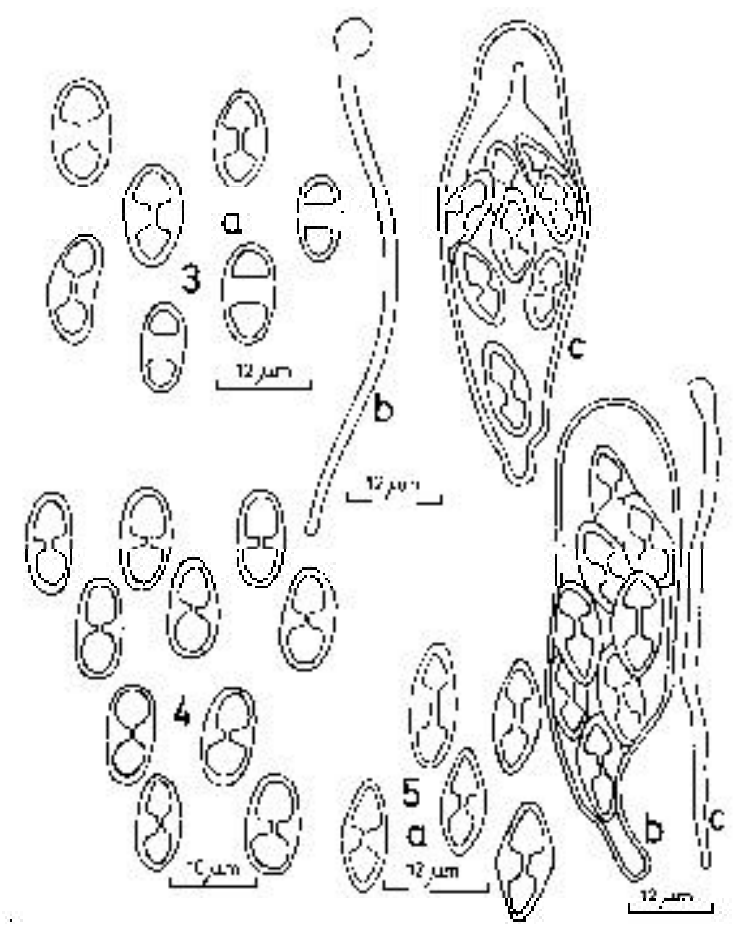

Figuras 3-5. Fig. 3. Caloplaca carphinea a) esporas, b) paráfisis y c) asco. Fig. 4. Caloplaca thallincola esporas. Fig. 5. Xanthoria ectaneoides a) esporas, b) asco y c) paráfisis.

Distribución MUNDiAL: En las costas de la región mediterránea (Poelt 1969); región mediterránea (Clauzade \& Roux 1985), Marruecos (Egea 1996).

Material estudiado: CHILE, VII Región, Prov. Talca. Constitución, Playa Los Gringos, 21-IV-2002. W. Torres, en rocas ubicadas más allá de la zona zona supralitoral, $\mathrm{N}^{\circ} 412$ (Herbario Universidad de Talca); Playa Vega Los Patos, 28-IV-2002. W. Torres $\mathrm{N}^{\circ} 411$ (Herbario Universidad de Talca).

Caloplaca thallincola (Wedd.) Du Rietz (Figs. 4 y 9) Talo crustáceo, continuo, con borde lobulado, anaranjado. Fotobionte: Trebouxia. Médula: blanca. Apotecios lecanorinos, anaranjados, de 0,2-0,4 mm de diámetro. Margen talino, permanente, anaranjado más oscuro que el disco; disco plano. Tecio incoloro, $80 \mu \mathrm{m}$ de altura. Epitecio anaranjado. Hipotecio incoloro. Paráfisis simples, algo abultadas en el extremo distal, a veces levemente ramificadas y pigmentadas. Ascos octosporadas. Esporas polariloculares, incoloras, elipsoidales, de $12-14$ x 4,8-6,4 $\mu \mathrm{m}$, con un tabique de 3,5-4 $\mu \mathrm{m}$ de ancho.
REACCIONES QUíMICAS: Cortex superior del talo, apotecio y epitecio $\mathrm{K}+$ púrpura.

DistRIBUCión MUNDiAL: Norte de Europa a la región mediterránea (Clauzade \& Roux 1985). Es común en el litoral Atlántico, de la Mancha, Mar del norte y el mar Báltico, rara vez ha sido encontrada en las costas mediterráneas (Roux 1987). Suroeste, oeste y Norte de Inglaterra, oeste de Europa, desde el norte de Francia a Escandinavia y Norteamérica (Purvis et al. 1992).

Material estudiado: CHILE, VII Región, Prov. Talca. Constitución, Playa Los Gringos, 21-IV- 2002, W. Torres, en zona intermareal y más raramente en la supralitoral. No 412 (Herbario Universidad de Talca).

Xanthoria ectaneoides (Nyl.) Zahlbr. (Figs. 5 a, b, c y 10)

Talo crustáceo, continuo, anaranjado, lobulado. Lóbulos dicotómicamente ramificados, ascendentes en los márgenes. Fotobionte: Trebouxia. Médula blanca. Apotecios lecanorinos, 0,08-0,4 mm de diámetro. Margen talino poco aparente, del mismo color del talo; disco plano, anaranjado. Tecio incoloro, 56-64 $\mu \mathrm{m}$ de altura. Epitecio anaranjado. Hipotecio incoloro. Ascos octosporados. Paráfisis simples, capitadas. Esporas polariloculares, incoloras, $12-17 \times 5,6-6,4 \mu \mathrm{m}$, septo de 2,4 $\mu \mathrm{m}$ de grosor.

REACCIONES Químicas: Cortex superior del talo y apotecio $\mathrm{K}+$ púrpura.

DistRIBUCión MUNDIAL : Israel (Galun \& Mukhtar 1996), Portugal (Hafellner 1995), centro y principalmente sur de Europa (Clauzade \& Roux 1985), Marruecos (Egea 1996), Gran Bretaña e Irlanda (Coppins 2002).

Material estudiado: CHILE, VII Región, Prov. Talca. Constitución, Playa Los Gringos, 21-IV-2002. W. Torres, en la zona supralitoral, $N^{\circ} 418$ (Herbario Universidad de Talca); Playa Vega Los Patos, 28-IV2002. W. Torres No419 (Herbario Universidad de Talca).

OBSERVACIONES: Es propia de ambientes marinos donde comparte su hábitat junto a Xanthoria parietina, con la cual es muy afín; pero se diferencia de ésta por presentar un talo fuertemente lobulado y ascendente por los márgenes. 

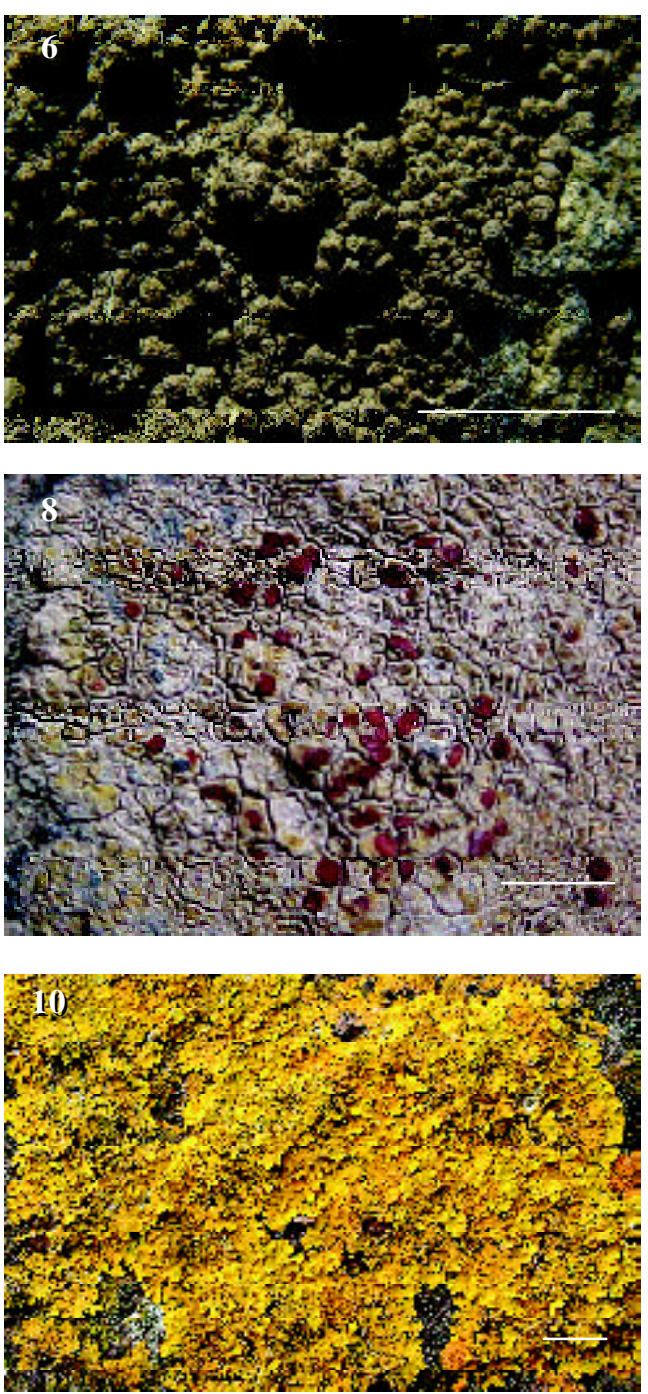

\section{AGRADECIMIENTOS}

Los autores agradecen a la DIUT de la Universidad de Talca el apoyo económico prestado en la realización de este trabajo. Al Sr. Pedro Arias en la confección de los dibujos a tinta.

\section{BIBLIOGRAFIA}

Aptroot, A. \& H.J.M. Sipman. 2001. New Hong Kong lichens, ascomycetes and lichenicolous fungi. Journal of the Hattori Botanical Laboratory 91: 317-343.

Archer, A.W. 1997. The lichen genus Pertusaria in Australia. Bibliotheca Lichenologica 69: 1 - 249.
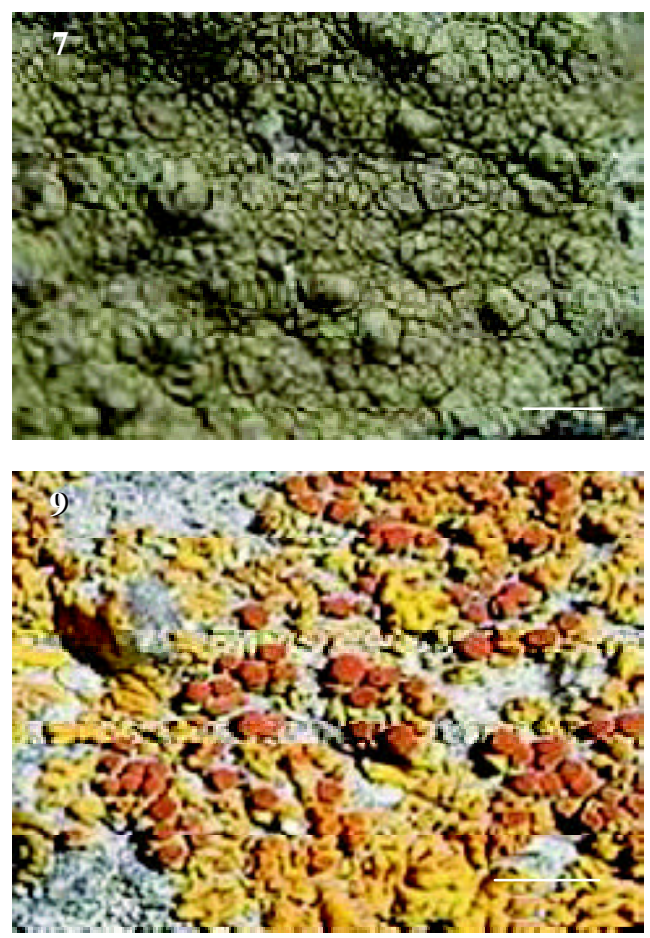

Figuras 6-10. Textura de los talos y los ascocarpos (apotecios y peritecios) de líquenes marinos chilenos. El trazo en blanco representa 1,5 mm. Fig. 6: Arthopyrenia saxicola talo y peritecio. Fig.7: Pertusaria rupicola talo y apotecio. Fig. 8: Caloplaca carphinea talo y apotecio. Fig 9: Caloplaca thallincola talo y apotecio. Fig. 10: Xanthoria ectaneoides talo.

Archer, A.W. \& J.A. Elix. 1993. Saxicolous species of Pertusaria (Lichens) common to New Zealand and Australia. New Zealand Journal of Botany 31: $111-116$

Clauzade, G. \& C. Roux. 1985. Likenoj de Okcidenta Europo. Ilustrita determininlibro. Bulletin de la Société Botanique du Centre-Ouest. Saint-Sulpicede-Royan - 17200 Royan (France). Nouvelle sérieNuméro Spécial 7. 893 pp.

Corneso, J. 2003. Sustentabilidad y medio ambiente. http:// lauca.usach.cl/ima/Que_es_EnviroInformatics.pdf Viewed: April 2005

CONAMA 2005. Recursos hídricos. Agua. hppt:// www.conama.cl/portal/1255/article-26359.html Viewed: April 2005.

Coppins, B.J. 2002. Checklist of lichens of Great Britain and Ireland. London, British Lichen Society. 45 pp. 
Egea, J.M. 1996. Catalogue of lichenized and lichenicolous fungi of Morocco. Bocconea 6: 19114

Esslinger, T.L. \& R.S. Egan. 1995. A Checklist of the Lichen-forming Lichenicolous, and Allied Fungi of the Continental United States and Canada. The Bryologist 98: 467-549.

Feuerer, T. 2004. Checklist of lichens and lichenicolous fungi of mainland Chile http.//www.biologie.unihamburg.de/checklists/chile_l.htm Viewed: April 2005 .

Follmann, G. 1964. Eine felsbewohnende Flechtengesellschaft der mittel- und nordchilenischen Küstenformation mit kennzeichnender Roccella portentosa (Mont.) Darb. Berichte der Deutschen Botanischen Gesellschaft 77: 262-276.

FollmanN, G. 1968a. Beobachtungen zur Verbreitung chilenischer Flechten. II Der Formenkreis von Roccella portentosa (Mont.) Darb. Nova Hedwigia 15: 333-343.

Follmann, G. 1968b. Felsbewohnende Arthoniaceen der chilenischen Pazifikküste. Willdenowia 4: 365382.

Follmann, G. 1995. On the impoverishment of the lichen flora and the retrogression of the lichen vegetation in coastal central and northern Chile during the last decades. Cryptogamic Botany 5: 224 - 231.

Galloway, D.J. \& W. Quilhot. 1998. Checklist of Chilean Lichen-forming and Lichenicolous Fungi. Gayana Botanica. 55: 111-185.

Galun, M. \& A. M uKhtAR. 1996. Checklist of the lichens of Israel. Israel Journal of Plant Science 44: 195218.

Hafellner, J. 1995. A new checklist of lichens and lichenicolous fungi of insular Laurimacaronesia including a lichenological bibliography for the area. Fritschiana 5: 1-132.

Malcolm, W.M. \& D.J. Galloway.1997. New Zealand Lichens: Checklist, key and glossary. Wellington, Museum of New Zealand Te Papa Tongarewa. 192 pp.

Poelt, J. 1969. Bestimmungsschlüssel europäischer Flechten. Verlag J. Cramer, Lehre. 757 pp.

Poelt, J. \& A. Vezda. 1977. Bestimmungsschlüssel europäischer Flechten. Ergänzungsheft I. Cramer, Vaduz. 158 pp.

Purvis, O.W., B.J. Coppins, D.L. Hawksworth, P.W. James \& D.M. Moore. 1992. The Lichen Flora of Great Britain and Ireland. Natural History Museum Publications \& British Lichen Society, London. $710 \mathrm{pp}$.

Roux, C. 1987. Lichens maritimes. En: Livre rouge des espèces marines et littorales menacées en France (eds. O. Gehu \& C. Roux.), pp. 327-345. MNHN, Paris.

Santelices, B. 1989. Algas marinas de Chile. Ediciones Universidad Católica de Chile, Santiago. 392 pp.

Santibánez, F, J. Uribe \& V. Marco. 1993. Atlas Agroclimático de Chile: regiones VI, VII, VIII y IX. Santiago de Chile. Ministerio de Agricultura. $99 \mathrm{pp}$.

Tehler, A. 1983. The genera Dirina and Roccellina (Roccellaceae). Opera Botanica 70: 1-86.

White, F.J. \& P.W. James. 1985. A new guide to microchemical techniques for the identification of lichen substances. British Lichen Society Bulletin 57: 1-41.

Wirth, V. 1980. Flechtenflora. Ökologische Kennzeichnung und Bestimmung der Flechten Südwestdeutsch-lands und angrenzender Gebiete. Verlag Eugen Ulmer Stuttgart. 478 pp. 